\title{
Adsorption characteristics of albumin on gold and titanium metals in Hanks' solution using EQCM
}

\author{
J.A. Lori ${ }^{\mathrm{a}, *}$ and T. Hanawa ${ }^{\mathrm{b}}$ \\ ${ }^{a}$ Department of Chemistry, Ahmadu Bello University, Zaria, Nigeria \\ ${ }^{\mathrm{b}}$ Biomaterials Research Team, National Institute for Materials Science, 1-2-1 Sengen, \\ Tsukuba 305-0047, Japan
}

\begin{abstract}
The adsorption of Bovine Serum Albumin (BSA) on gold and titanium surfaces in Hanks' solution was monitored using the electrochemical quartz crystal microbalance (EQCM) technique. The changes in mass and open circuit potential at rest potential and mass and current at constant potentials were measured before and after the introduction of BSA.

The mass of BSA adsorbed on titanium reached a steady value within one hour, while the albumin adsorbed on gold continued to increase. The amount of BSA adsorbed by titanium increased with concentration and stabilized at $0.8 \mathrm{mg} / \mathrm{ml}$ while that on gold did not stabilize. This indicates a monolayer of the protein on titanium and multi-layer on gold. The adsorption of BSA on gold and titanium was accelerated by the charge of potential.

The time before stabilizing the adsorption-desorption phenomena also increased with the BSA concentration.
\end{abstract}

Keywords: Quartz crystal microbalance, EQCM, adsorption, gold, titanium, bovine serum albumin

\section{Introduction}

The interaction between metal surfaces and tissues is a complex matter. The precise nature of the adsorption of proteins onto these artificial surfaces (e.g., titanium and gold) by the host system is important.

The initial events that occur upon contact between the metals and tissues are also important and may themselves have an influence on subsequent events [1]. The understanding of the adsorption and cooperative mechanisms of amino acids and proteins on surfaces is of importance in research of biomaterials [1,2] and cell adhesion [3]. Surgical metal implants in humans were primarily developed to function as prosthetic devices either for the replacement of damaged parts that cannot be replaced through treatment or fixation devices for holding bone parts in place until healing is established. The disadvantage of inadequate strength and inertness exhibited by earlier materials has resulted in the adoption of titanium and titanium alloys, stainless steel AISI Type 316L (SS 316L) and cobalt-chromium-molybdenum (Co-Cr-Mo) alloy for implants in man [4-6].

It is important that adsorption of amino acids and proteins onto different materials used as artificial implants be studied, since the adsorption of proteins is one of the first events that occurs when the artificial implant is brought in contact with human tissue or body fluids [7].

\footnotetext{
${ }^{*}$ Corresponding author. Tel./Fax: +234 62 217219; E-mail: josephlori@yahoo.co.uk.
} 
Protein adsorption has been thoroughly studied by the use of different techniques [8]. Understanding the factors governing protein adsorption on artificial materials and their displacement from the interface should provide information that will be useful for the development of materials exhibiting improved biocompatibility.

Interfacial processes at electrode surface require utilization of sophisticated techniques in order to obtain detailed information on structure and composition of interfaces. Quartz crystal microbalance (QCM) is one of the novel methods applied in the field of electrochemical interfaces. The QCM is an ultrasensitive weighing device and consists of a thin disk of quartz crystal with thin electrodes deposited on each side. Any mass added to or removed from the electrode surface induces a shift in the resonant frequency of oscillation of the crystal, which is related to the mass change [9]. Application of QCM to reactions in an electrochemical cell is referred to as electrochemical quartz crystal microbalance $(\mathrm{EQCM})$. We have recently reported the characterization of glycine on gold and titanium using the EQCM technique [10].

We report the study of the adsorption of BSA in Hanks' solution, on gold and titanium as characterized using the EQCM technique. The changes in mass and potential at rest potential and mass and current at a constant potential were measured before and after BSA was injected into the electrochemical environment.

\section{Experimental methods}

The authors recently described the theory of quartz crystal microbalance and calculation methods as employed in this study [10].

\subsection{Specimens}

Gold and titanium were sputter-deposited on the quartz crystal disk. The quartz crystal used was an AT-cut disk of $1.3 \mathrm{~cm}$ diameter and of 6-MHz nominal oscillation frequency. Exposed area of the metals to the solution was $1.3267 \mathrm{~cm}^{2}$.

\subsection{Solutions}

The ion concentrations of Hanks' solutions are summarized in Table 1. This solution without organic species has ion concentrations similar to those of extra cellular fluid and was employed as an electrolyte in this study. The solution was bubbled by $4 \% \mathrm{O}_{2} / \mathrm{N}_{2}$ gas for at least $1,200 \mathrm{~s} .4 \% \mathrm{O}_{2} / \mathrm{N}_{2}$ gas was employed, because the partial pressure of oxygen in the gas is almost the same as that in body fluid. The Hanks' solution had a pH of 7.4 just after preparation. All chemicals used were analytical grade (99$100 \%$ purity, Kanto Chemical Co., Japan). Stock solution for injection was prepared from bovine serum albumin (>96\% pure, Sigma Chemical Co., MO, USA) as a concentration of $4 \mathrm{mg} / \mathrm{ml}$ in the Hanks' solution. During the experiments, the reaction solution was maintained at a temperature of $310 \mathrm{~K}$.

\subsection{QCM analysis under rest potential}

An EQCM controller Model HQ 101B connected to an electrochemical analyses system, Model HZ-3000 (Hokuto Denko, Japan) was used to monitor the frequency shift, $\Delta f$. The counter and reference electrodes were platinum and saturated calomel (SCE), respectively. The gold or titanium quartz crystal completed the setup as working electrodes in each case. 
Table 1

\begin{tabular}{lc}
\multicolumn{2}{c}{ Ion concentrations of Hanks' solution } \\
\hline Ion & Concentration $\left(\mathrm{moll}^{-1}\right)$ \\
\hline $\mathrm{Na}^{+}$ & $1.42 \times 10^{-1}$ \\
$\mathrm{~K}^{+}$ & $5.81 \times 10^{-3}$ \\
$\mathrm{Mg}^{2+}$ & $8.11 \times 10^{-4}$ \\
$\mathrm{Ca}^{2+}$ & $1.26 \times 10^{-3}$ \\
$\mathrm{Cl}^{-}$ & $1.45 \times 10^{-1}$ \\
$\mathrm{HPO}_{4}^{2-}$ & $7.78 \times 10^{-4}$ \\
$\mathrm{SO}_{4}^{2-}$ & $8.11 \times 10^{-4}$ \\
$\mathrm{CO}_{3}^{2-}$ & $4.17 \times 10^{-3}$ \\
\hline
\end{tabular}

The $\Delta f$ of the specimen was allowed to stabilize before injecting the BSA solution with a syringe. The resultant concentrations of BSA after injection in this study were $0.1,0.2,0.4,0.6,0.8$ and $1.0 \mathrm{mg} / \mathrm{ml}$. The $\Delta f$ and change in open circuit potential, $E_{\text {open }}$, of titanium and then gold were measured by the EQCM.

\subsection{QCM analysis with constant potential}

Under similar experimental conditions, the experimental procedure for the rest potential experiment was repeated at constant potentials of $0 \mathrm{~V}, 0.5 \mathrm{~V}$ and $0.75 \mathrm{~V}$ vs. SCE while monitoring $\Delta f$ and changes in the current generated, $\Delta I$.

\section{Results and discussion}

\subsection{Viscosity and density before and after injection}

The changes in viscosities and densities of the resultant solution after the injection of BSA stock solution into the Hanks' solution were infinitesimal in the concentrations studied. Similar observation has been reported previously [10]. A suggestion that the steep change in frequency upon injection of the protein may be as a result of change in viscosity is therefore unlikely. If this is even so, then, the frequency is able to quickly recover within split seconds and the resultant solution had no significant change in viscosity. The $\Delta m$ for each electrode was therefore calculated without the viscosity and density variables as previously reported [10].

\subsection{QCM analysis with rest potential}

The time transient of $\Delta f$ under rest potential are simply illustrated in Fig. 1A. Following the injection of the BSA solution, $\Delta f$ momentarily decreased then gradually increased, and eventually stabilized. We define the value of the first momentary decrease of $\Delta f$ as $\Delta f_{1}$ and the resultant decrease of $\Delta f$ after gradual increase as $\Delta f_{2}$. The $\Delta f_{1}$ was caused by the momentary adsorption of BSA just after injection, followed by a desorption phase and at equilibrium a resultant adsorption of BSA is achieved which is depicted by $\Delta f_{2}$, which remains constant until the end of the experiment. This pattern is same for both gold and titanium electrodes. This phenomena is similar to that previously reported for glycine under similar conditions [10]. However, the $\Delta E$ of the two electrodes followed differing patterns as shown in 


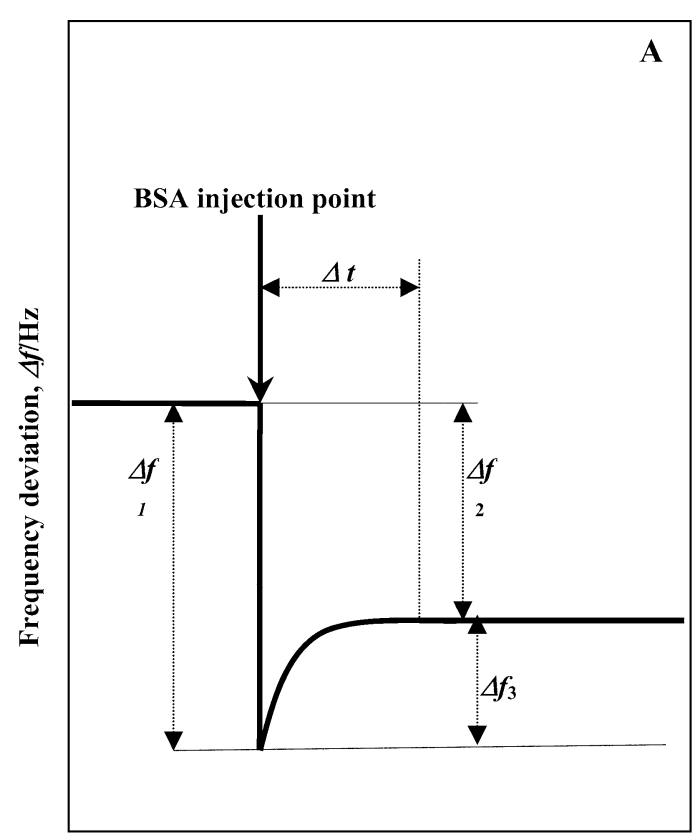

Time, $t / \mathbf{s}$

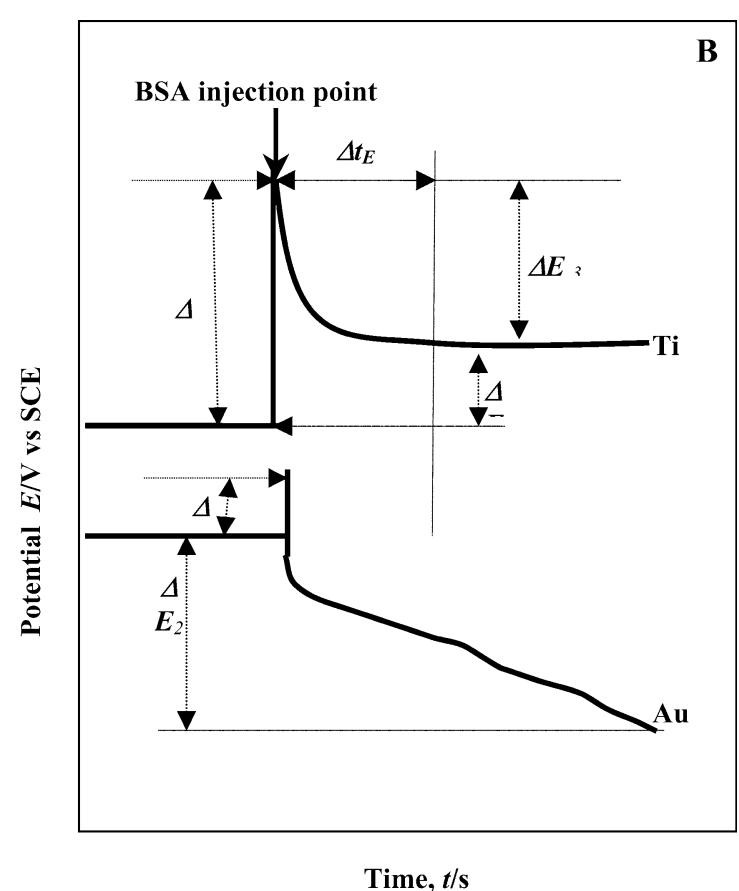

Fig. 1. Time transient of resonant frequency (A) and open circuit potential (B) under rest potential before and after the injection of BSA.

Fig. 1B. The gold electrode showed abrupt decrease just after injection of BSA, with a minimum at $\Delta E_{1}$, and continuously but more slowly increased, without equilibrating until the end of the experiment. This phenomenon is different from that reported for glycine [10]. On the other hand, titanium showed first a sharp decrease followed by a slight increase before equilibrating. Titanium behavior with BSA is similar to that reported for glycine [10]. However, the profiling is slower for BSA than for glycine, obviously due to molecular weight factor.

The $\Delta E$ were negative in gold and positive for titanium. Time for establishing equilibrium $\Delta E_{3}$ is expressed as $\Delta t_{E}$. Equilibrium was not established for the $\Delta E$ of gold.

The $\Delta m_{1}$ and $\Delta m_{2}$ of gold and titanium calculated from $\Delta f_{1}$ and $\Delta f_{2}$ as a function of concentration of BSA are shown in Figs 2A and 2B, respectively. Both $\Delta m_{1}$ and $\Delta m_{2}$ in gold was larger than those in titanium at all concentrations of BSA. This is directly the opposite of what we reported for glycine previously [10].

Metal surfaces are, in general, hydrophilic, e.g., titanium. However, gold, to a greater extent have less hydrophilic surfaces. It has been reported [12] that the hydrophobicity of metals surface might be considered an important factor in determining the protein adsorption characteristics to metal surfaces. It might be reasonable to suspect from Figs 1 and 2 that a monolayer of BSA was adsorbed on to titanium while a multi-layer of BSA was adsorbed on the gold electrode.

The potential of the gold electrode did not equilibrate throughout the duration of the experimental (about 6 hours) and therefore the $E_{3}$ value for the gold electrode was not estimated. $E_{2}$ for the gold electrode was taken from the final value of $E$ at the end of the experiment. The $E_{\text {open }}$ of gold and titanium just before injection of BSA were 29.4 to $58.0 \mathrm{mV}$ and -195.6 to $-240.0 \mathrm{mV}$, respectively. Relative to their $E_{\text {open }}$ the changes in potential of gold were negative while those of titanium were positive. These are presented in absolute values of potential as a function of $c$ in Figs $3 \mathrm{~A}$ and $3 \mathrm{~B}$. The $E_{2}$ of gold were 

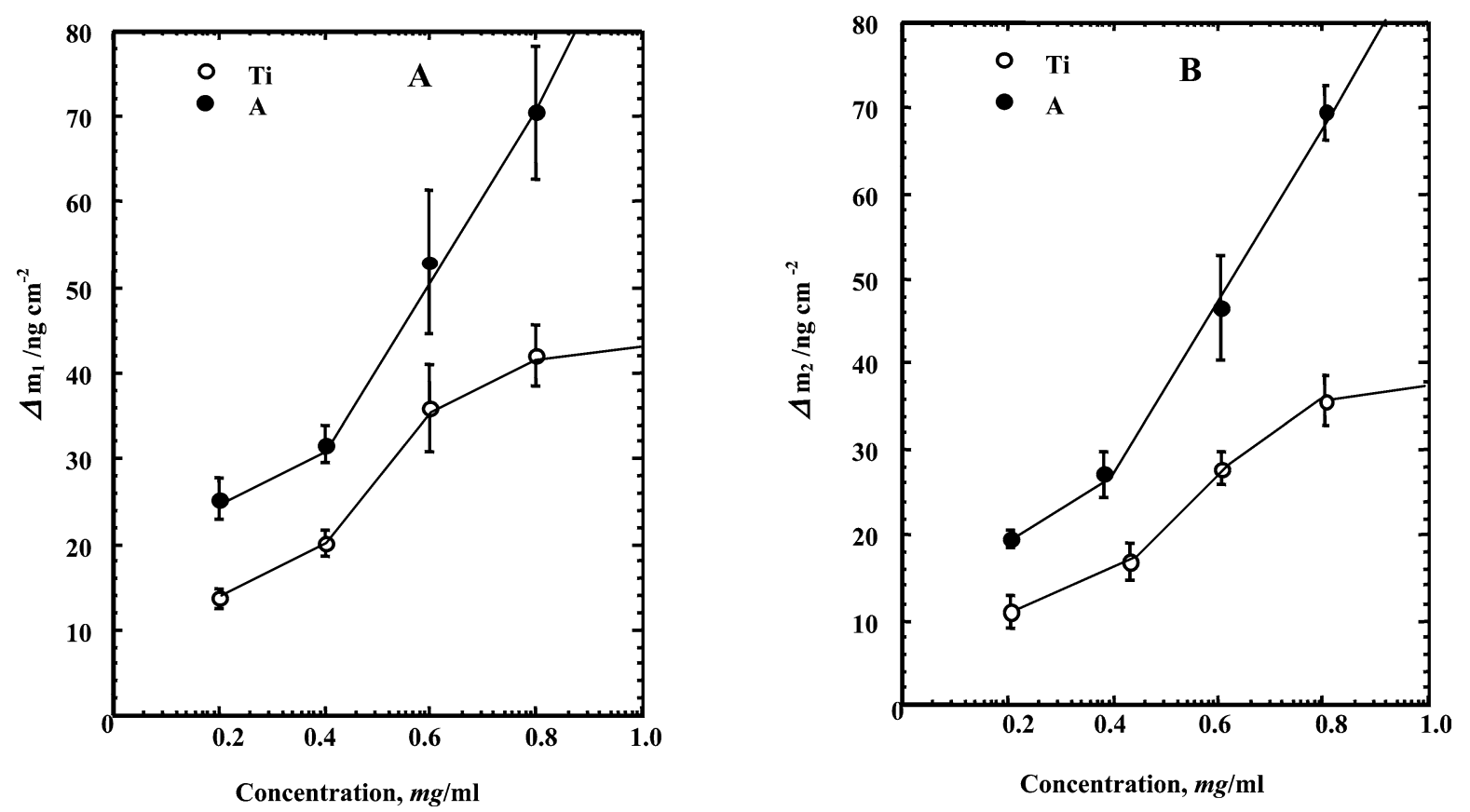

Fig. 2. Mass changes, $\Delta m_{1}$ (A) and $\Delta m_{2}$ (B) of gold and titanium calculated from $\Delta f_{1}$ and $\Delta f_{2}$ defined in Fig. $1 \mathrm{~A}$ under rest potential.
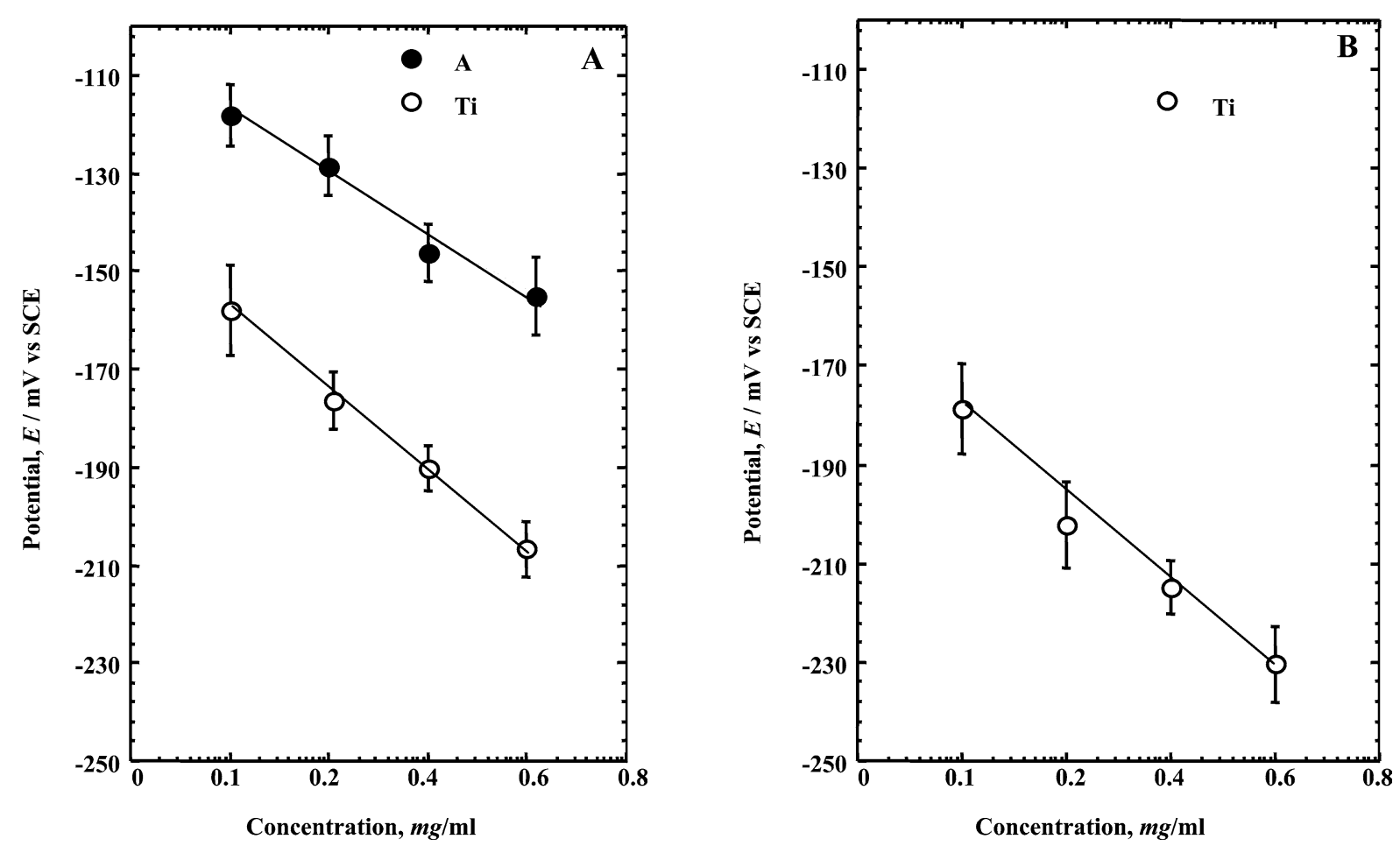

Fig. 3. Electrochemical changes, $E_{2}(\mathrm{~A})$ and $E_{3}(\mathrm{~B})$ defined in Fig. 1B of gold and titanium as a function of concentration of BSA. 


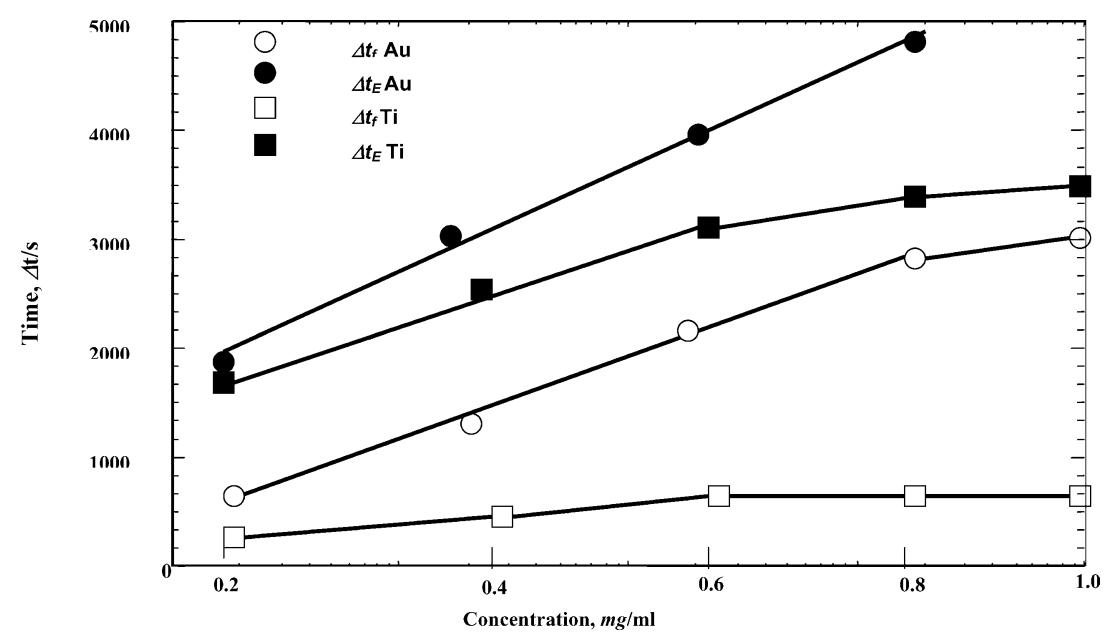

Fig. 4. Time before stabilizing the frequency, $\Delta t_{f}$ (Fig. 1A), and time before stabilizing the potential, $\Delta t_{E}$ (Fig. 1B), of gold and titanium as a function of concentration of BSA under rest potential.

larger than those of titanium, however the net change in potential, $\Delta E$ for each metal electrode relative to their $E_{\text {open }}$ show that the titanium exhibits a positive change in potential higher than the negative values of gold. That is, gold may be corroded by the adsorption of BSA, whereas the adsorption layer of BSA on titanium works as a surface film. This is similar to the results reported for these electrodes in the presence of glycine.

The $\Delta t_{f}$ and $\Delta t_{E}$ in gold and titanium as a function of BSA concentration are shown in Fig. 4. The $\Delta t_{f}$ is the time taken from injection of BSA and the point at which $\Delta f$ begins to stabilize (Fig. 1A) and $\Delta t_{E}$ (Fig. 1B) is the time from injection to the stabilization of $E$. The $\Delta t_{f}$ and $\Delta t_{E}$ increase proportionally with increase in the BSA concentration, indicating the adsorption phenomena is dependent on time and concentration.

\subsection{QCM analysis with constant potential}

Figure 5 shows the current generated by the adsorption of BSA on gold and titanium under constant potential of $0.5 \mathrm{~V}$ vs SCE as a function of BSA concentration. For all concentration of BSA studied, the gold electrode exhibited a phenomena of a sharp current generation immediate upon injection of BSA that never equilibrated in the duration of the experiment. This trend was observed for gold at all concentration studied under the constant potential of $0.5 \mathrm{~V}$ vs SCE. This shows that gold may corrode in the presence of BSA under applied potential. Current generated by the adsorption of BSA on titanium under similar condition equilibrated quickly. Again this is indicative of a single uniform monolayer of BSA on the titanium surface.

\subsection{Factors influencing the adsorption of BSA}

The amount of BSA adsorbed on gold and titanium and the time until finishing the adsorption increased with the increase of concentration of BSA because of the molecular size of BSA.

More BSA is adsorbed on the metals as the concentration of BSA in the resultant solution increased. However, the resultant adsorbed BSA in relation to the initial mass change, increased as the concentra- 


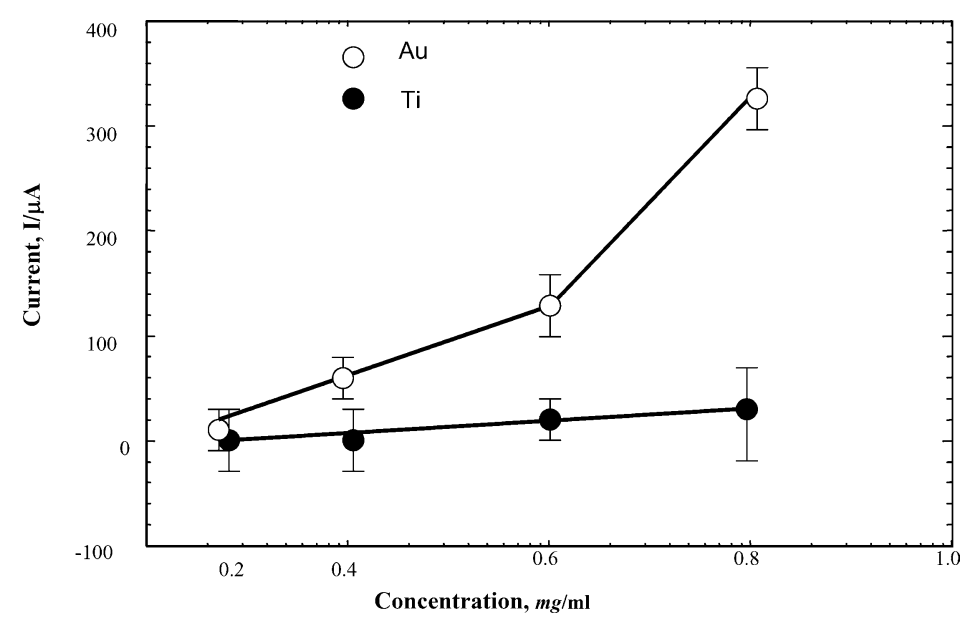

Fig. 5. The $\Delta m_{1}$ (A) and $\Delta m_{2}$ (B) of gold and titanium calculated from $\Delta f_{1}$ and $\Delta f_{2}$ with as a function of concentration of BSA under constant potential.

tion of the BSA in the resultant solution decreases. This suggests that BSA may be saturated in the range of concentrations studied.

More BSA was adsorbed faster on gold than on titanium at rest potential unlike when glycine was studied recently in a similar system. Gold surface in aqueous solutions is not oxidized, but slightly hydrated.

Metal surfaces carry a positive charge and proteins (e.g., BSA) with a pH lower than 7.4 are generally negatively charged at physiological $\mathrm{pH}$, it is possible that $\mathrm{BSA}$ may be adsorbed onto the metal surfaces by electrostatic forces as previously reported [8]. In the constant potential experiments, gold adsorbs more BSA momentarily, than titanium at the same potential. Also, the resultant amount of BSA adsorbed on titanium was far less than that on gold. This indicates that electric force on gold was stronger than on titanium. Gold adsorbed higher levels of BSA and have more noble standard electrode potential. The more noble the standard electrode potential, the greater the positive character of the metal and therefore, the stronger the electrostatic attraction for negatively charged molecules.

The $E_{\text {open }}$ of gold and titanium were changed by the adsorption of BSA. The $E_{\text {open }}$ of gold decreased by the adsorption of BSA. This indicates that gold is apparently corroded by the adsorption of BSA. On the contrary, the $E_{\text {open }}$ of titanium increased with the increase of the amount of adsorbed BSA, indicating that titanium did not corrode and adsorption layer was apparently more uniform with increasing concentration of BSA.

\section{Conclusions}

The amount of BSA adsorbed on gold and titanium and the time before stabilization of the adsorption increased with the increase of concentration of BSA as reported for glycine [10]. More BSA was adsorbed faster on gold than on titanium unlike with glycine. The adsorption was accelerated by the charge of potential as with glycine. The attractive force for the adsorption of BSA to metal surface is the electric charge of the metal surface rather than the electrostatic attractive force. The adsorption of amino acid (glycine) has a totally different phenomena from the adsorption of protein (BSA) on the gold 
electrode. The QCM technique is useful to characterize the adsorption phenomena of proteins to metal surface.

\section{Acknowledgements}

We acknowledge the Japan Institute of Science and Technology (JISTEC), STA Research Fellowship granted the first author from which this work was funded.

\section{References}

[1] J.D. Andrade and V. Hlady, Plasma protein adsorption: the big twelve, Ann. N. Y. Acad. Sci. 516(1) (1987), 158-172.

[2] R.E. Baier, Conditioning surfaces to suit the biomedical environment: Recent progress, J. Biomechan. Eng. 104 (1982), 257-271.

[3] B. Ivarsson and I. Lundstrom, Self-assembled organic monolayer: Model systems for studying adsorption of protein at surfaces, Crit. Rev. Biocompat. 2 (1986), 1-96.

[4] J.A. Lori and T. Hanawa, Characterization of adsorption of glycine on gold and titanium electrodes using electrochemical quartz crystal microbalance, Corr. Sci. 43(11) (2001), 2111-2120.

[5] G. Sauerbrey, Verwendung von Schwingquarzen zur Wagung dunner Schichten und zur Microwagang, Z. Phys. 155 (1959), 206-212.

[6] P.N. Sawyer and S. Srinivasan, Synthetics and implants, in: Medical Engineering, Ch.D. Ray, ed., Year Book Medical Publishers, Chicago, Illinois, 1994, pp. 1099-1101.

[7] J.M. Schakenraad and H.J. Busscher, 1989, Cell-polymer interactions: The influence of protein adsorption, Coll. Surf. 42(2) (1989), 331-343.

[8] J.E. Sundgren, P. Bodo, B. Ivarsson and I. Lundstrom, Protein adsorption and fibroblast adhesion on irradiated polysiloxane surfaces, J. Coll. Interface Sci. 113 (1986), 530-537.

[9] D.F. Williams and R.G. Bagnall, Biocompatibility, in: Fundamental Aspects of Biocompatibility, Vol. II, D.F. Williams, ed., CRC Press, Boca Raton, Florida, 1981, pp. 113.

[10] J.A. Lori and T. Hanawa, Characterization of adsorption of glycine on gold and titanium electrodes using electrochemical quartz crystal microbalance, Corrosion Science 43(11) (2001), 2111-2120. 


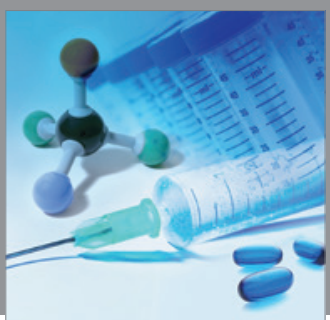

International Journal of

Medicinal Chemistry

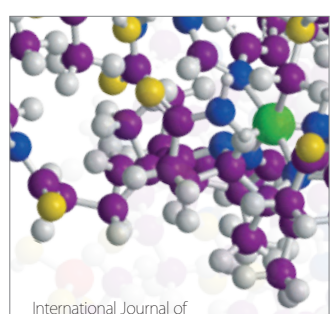

Carbohydrate Chemistry

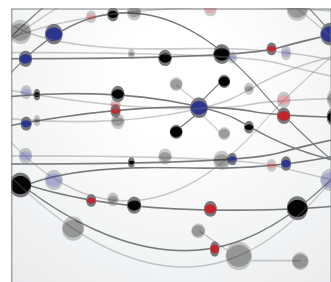

The Scientific World Journal
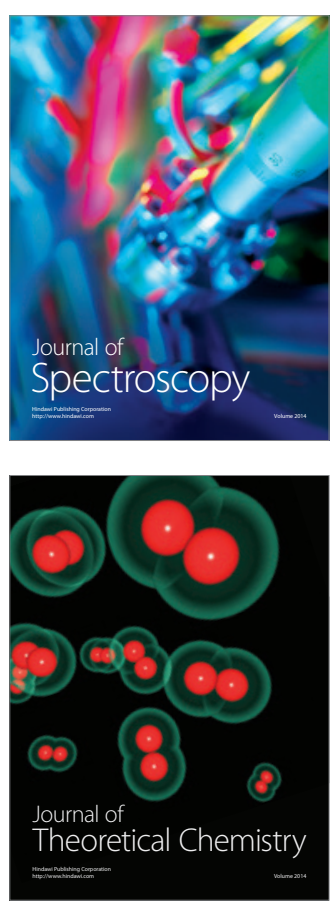
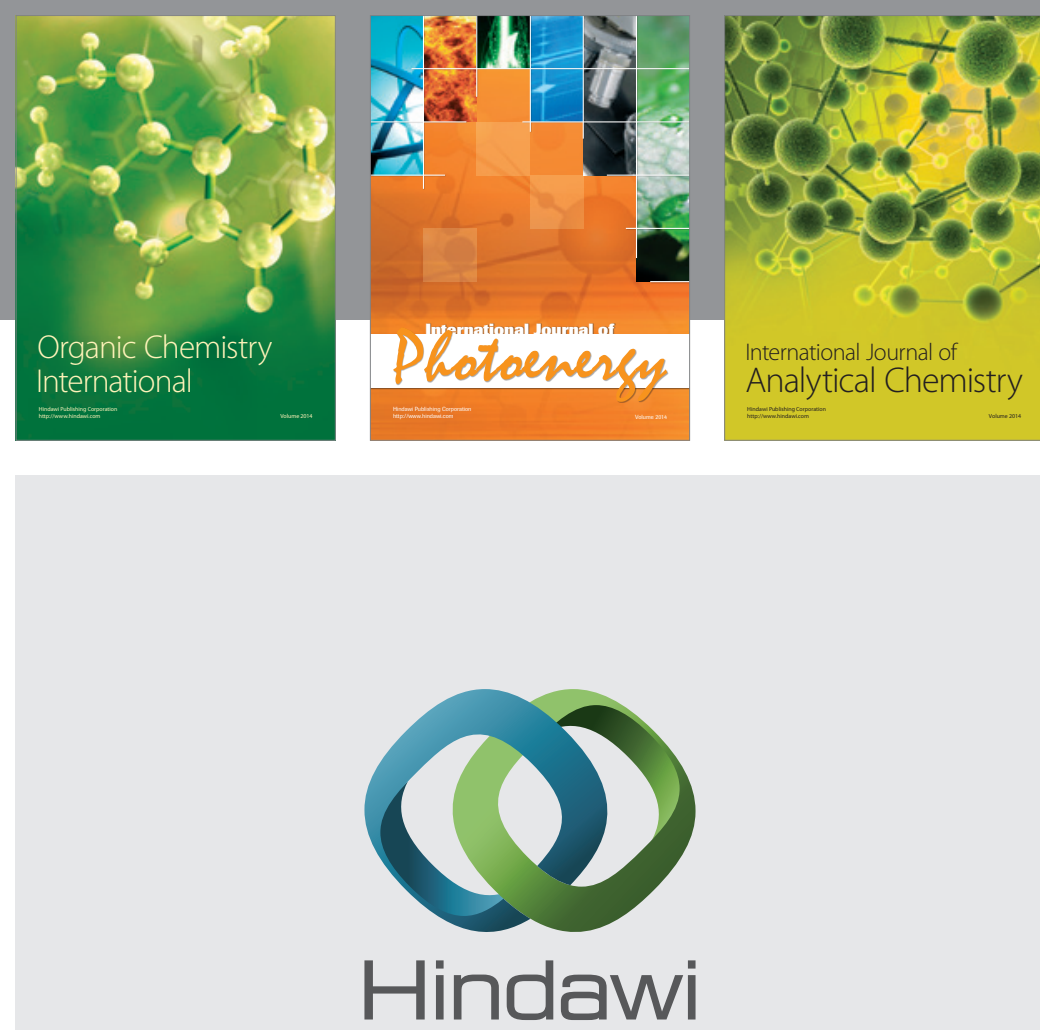

Submit your manuscripts at

http://www.hindawi.com
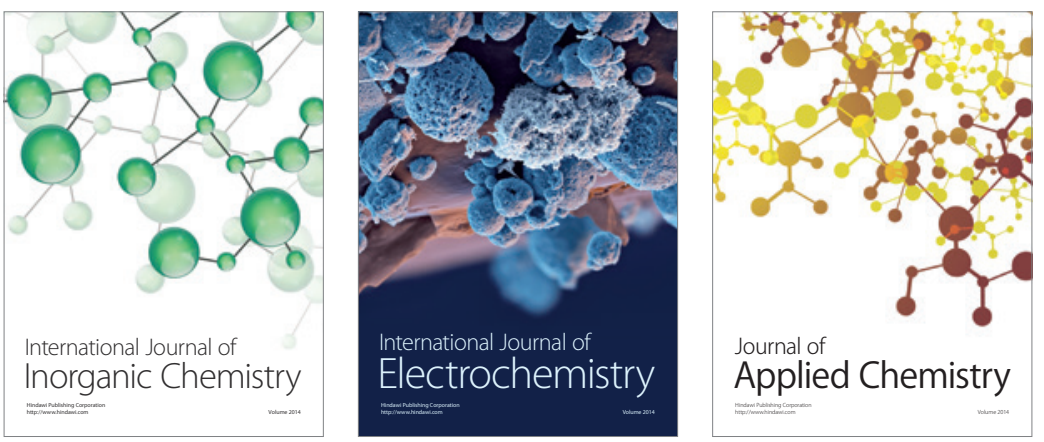

Journal of

Applied Chemistry
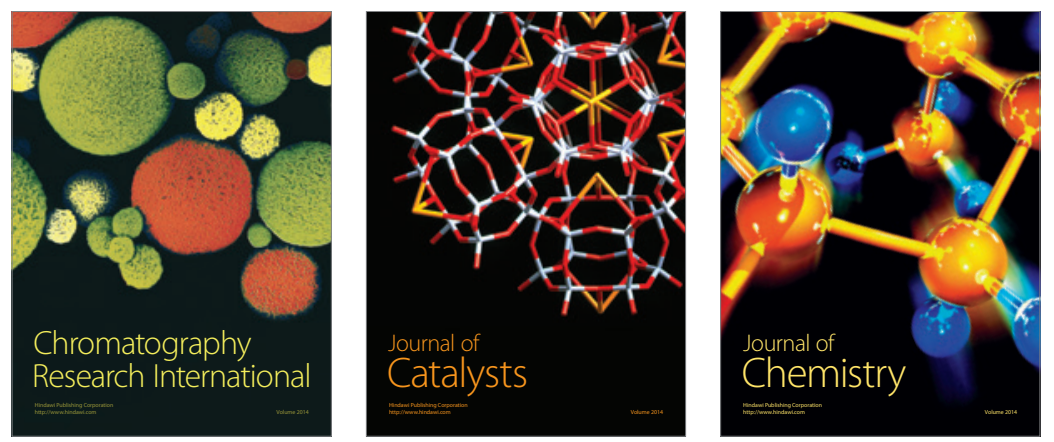
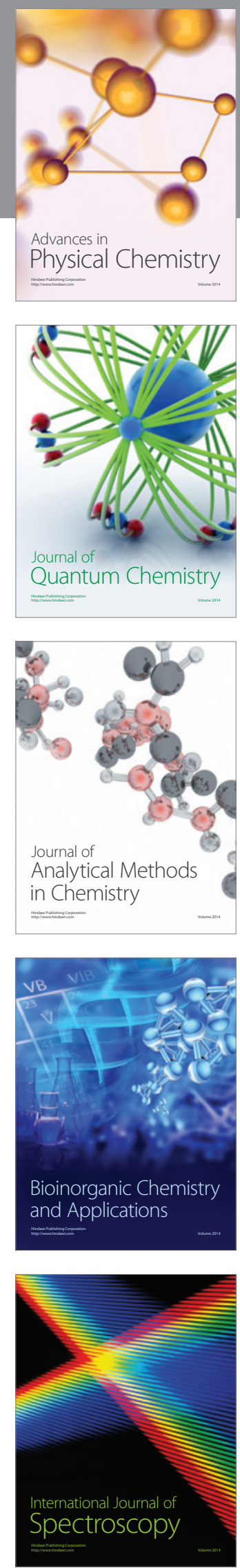